\title{
Two-Dimensional and Three-Dimensional Imaging of the In Vivo Lung: Combining Spiral Computed Tomography With Multiplanar and Volumetric Rendering Techniques
}

\author{
Janet E. Kuhlman, Derek R. Ney, and Elliot K. Fishman
}

\begin{abstract}
We applied multiplanar techniques and a modified version of our volumetric rendering program for threedimensional imaging to single-breath hold spiral computed tomography (CT) datasets to generate two- and three-dimensional (2-D and 3-D) images of the in vivo lung. We report details of the combined 2-D/3-D spiral CT technique along with three representative cases from our initial experience.

Copyright 1994 by W.B. Saunders Company
\end{abstract}

KEY WORDS: spiral computed tomography, threedimensional imaging, multiplanar reconstruction.

$\mathbf{T}$

WO-DIMENSIONAL (2-D) and threedimensional (3-D) imaging of the in vivo lung has been limited to date by respiratory motion. Conventional slice-by-slice computed tomography (CT), a stepwise technique, often results in variations in depth of inspiration and patient motion that significantly degrade the quality of reconstructed images. Spiral volumetric CT, on the other hand, is essentially a gapless technique ${ }^{1-4}$ with a number of advantages for 2-D and 3-D imaging. Volumetric acquisition eliminates discontinuities between slices and the examination is performed during a single-breath-hold maneuver that significantly reduces respiratory motion.

Because of these advantages, we applied multiplanar and volumetric rendering techniques to single-breath-hold spiral CT datasets to generate 2-D and 3-D images of the in vivo lung. The combined technique is compared with conventional CT and spiral CT alone, and is the subject of this article.

\section{MATERIALS AND METHODS}

Single-breath-hold spiral CT datasets of the lung were obtained in 30 patients with known

From The Russell H. Morgan Department of Radiology and Radiological Science, The Johns Hopkins Medical Institutions, Baltimore, $M D$.

Address reprint requests to Janet $E$. Kuhlman, $M D$, The Department of Radiology, The Johns Hopkins Hospital, $600 \mathrm{~N}$ Wolfe St, Baltimore, MD 21205.

Copyright $\odot 1994$ by W.B. Saunders Company

0897-1889/94/0701-0001\$03.00/0 pulmonary pathology and/or previous conventional CT scans of the chest for comparison. Spiral CT datasets were obtained using a continuously rotating Siemens Somatom Plus scanner (Siemens Medical Systems, Iselin, NJ) capable of generating up to 24 consecutive 1 -second scans and one of the following protocols: (1) slice acquisition thickness $8 \mathrm{~mm}$, table speed $8 \mathrm{~mm} / \mathrm{sec}$, and slice reconstruction every $4 \mathrm{~mm}$ with standard reconstruction algorithm or (2) slice acquisition thickness $4 \mathrm{~mm}$, table speed $4 \mathrm{~mm} / \mathrm{sec}$, and slice reconstruction every $2 \mathrm{~mm}$ with high-resolution algorithm. Typical scanning parameters were $165 \mathrm{~mA}$ and $120 \mathrm{kV}(\mathrm{p})$. The Spiral CT data is acquired orthogonal to the table translation axis.

Spiral CT data was transferred using halfinch magnetic tape or via Ethernet to a freestanding unit consisting of a Sun 4/330 workstation (Sun Microsystems, Mountain View, CA) and a Pixar I image computer (Vicom, San Rafael, CA) where multiplanar and volumetric rendering techniques designed for lung analysis were applied. The Sun/Pixar system has been used previously to generate high quality 2-D and 3-D reconstructions of musculoskeletal structures. ${ }^{5,6}$

Volumetric rendering consists of a three-step process: volume formation, classification, and projection. Three-dimensional volumetric rendering poses special problems requiring a new classification scheme when applied to the in vivo lung. Classification involves assigning a color and opacity to each voxel in the CT dataset (Fig 1). The lung is composed of vessels, bronchi, and mostly air. Thus, partial volume effects of the lung are prominent but allow classification of lung structures primarily based on size. For example, a voxel containing a large vessel might read 40 to 80 Hounsfield units $(\mathrm{Hu})$ and would be assigned a particular color and opacity. A voxel containing a small vessel and $90 \%$ air might read $-900 \mathrm{Hu}$ and would be assigned a different color and opacity. Larger structures 

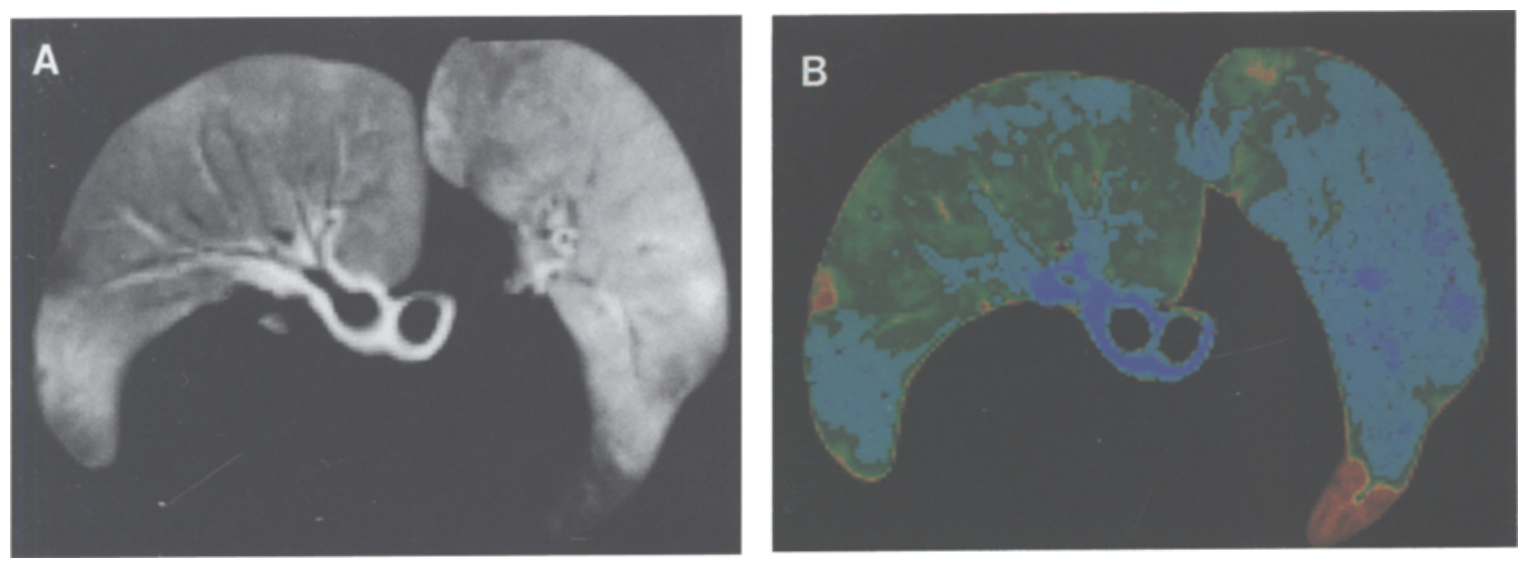

Fig 1. Classification scheme used for volumetric rendering of lung tissue. Color and opacity are assigned to lung structures based primarily on their size and CT attenuation. (A) Original CT scan of lung specimen. (B) Color representation of classified slice to be used for volumetric rendering. Dark blue represents large structures with relatively high $C T$ attenuation such as the walls the major bronchi. Light blue represents medium size or medium density structures such as lung parenchyma of medium CT attenuation. Green and red represent smaller structures or lower density areas. The red areas are portions of the lung showing the lowest CT attenuation on the original CT scan.

are assigned a higher opacity than smaller structures because smaller structures tend to be in the periphery of the lung and would mask the larger structures if they were made opaque.

Once classified, the spiral CT data is projected in a series of 64 to 72 separate images viewed from different angles around the vertical axis of the lung. The projections are formed using a gradient shading algorithm and the views are displayed as a videoloop, in which the lung appears to be spinning around its vertical axis.

The spiral CI data sets were also examined using a multiplanar reconstruction program (2D/3D Orthotool software, Body CT Imaging Laboratory, Department of Radiology, The Johns Hopkins Medical Institutions, Baltimore, MD) that provides interactive, simultaneous display of coronal and sagittal reformatted images along with the original transaxial scans. A bicubic interpolation scheme is used to produce the reformatted images. ${ }^{7}$ Average time required to generate both the 2-D multiplanar reconstructions and the 3-D lung images using the Sun/ Pixar system was 15 minutes per case.

All 2-D/3-D spiral CI images were subjectively compared with available conventional CT scans and the spiral CT scans alone to assess the relative quality and accuracy of the 2-D and 3-D images.

\section{CASE REPORTS}

\section{Case 1}

A 50-year-old woman with a history of resected squamous cell cancer of the larynx was being followed up for a small pulmonary nodule. An axial reconstructed image from a spiral CT dataset (Fig 2A) and multiplanar reconstructed images of the lung in the sagittal and coronal planes are shown in Fig $2 \mathrm{~B}, \mathrm{C}$. The nodule as well as the surrounding parenchymal emphysema are faithfully reproduced in the reconstructed images. Note the definition, without motion degradation, of the major airways on the coronal reconstruction (Fig 2C). Threedimensional images of the lung created by applying volumetric rendering techniques to spiral CI data are shown in Fig 2D, E. Lung parenchyma, bronchovascular structures, major airways, and the small nodule are displayed without significant respiratory artifacts or image distortion. (Protocol 2: slice acquisition thickness $4 \mathrm{~mm}$, table speed $4 \mathrm{~mm} / \mathrm{sec}$, and slice reconstruction every $2 \mathrm{~mm}$ with high-resolution algorithm.)

\section{Case 2}

A 39-year-old woman receiving corticosteroids for a renal transplant was noted to have a 

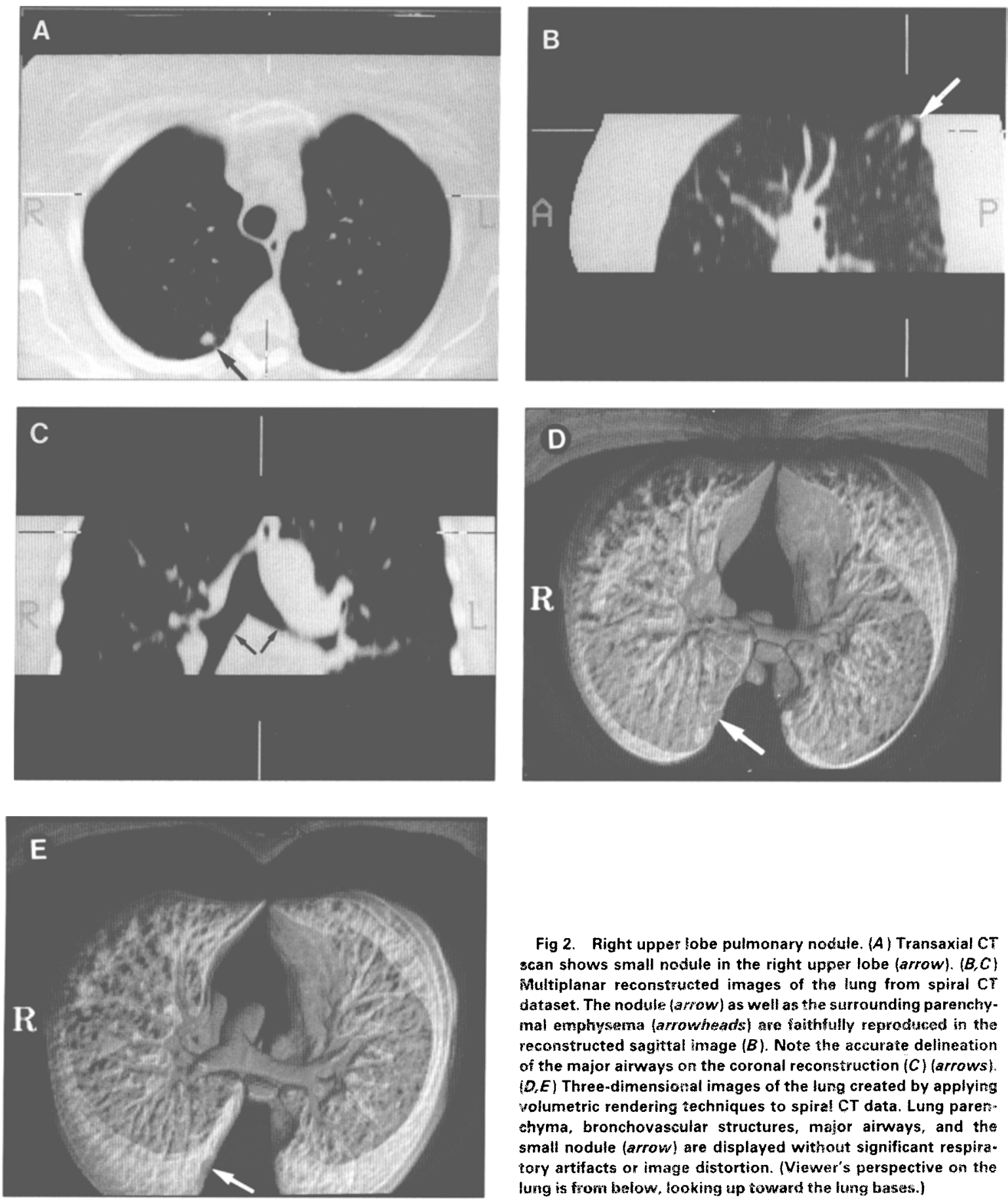

Fig 2. Right upper lobe pulmonary nodule. $(A)$ Transaxial $C T$ scan shows small nodule in the right upper lobe (arrow). $(B, C)$ Multiplanar reconstructed images of the lung from spiral $\mathrm{CT}$ dataset. The nodule (arrow) as well as the surrounding parenchymal emphysema (arrowheads) are laithfully reproduced in the reconstructed sagittal image $(B)$. Note the accurate delineation of the major airways on the coronal reconstruction (C) (arrows). $(D, E)$ Three-dimensional images of the lung created by applying volumetric rendering iechniques to spira! CT data. Lung parerchyma, bronchovascular structures, major airways, and the small nodule (arrow) are displayed without significant respira. tory artifacts or image distortion. (Viewer's perspective on the lung is from below, looking up toward the lung bases.)

nodule on chest radiograph. A transaxial view generated from spiral CT data shows a nodule with a feeding artery and draining vein compatible with a pulmonary arteriovenous malforma-

tion (Fig 3A). Volumetric rendering of the lung using spiral CT data shows the pulmonary arteriovenous malformation in a 3-D format (Fig 3B). (Protocol 1: slice acquisition thickness 

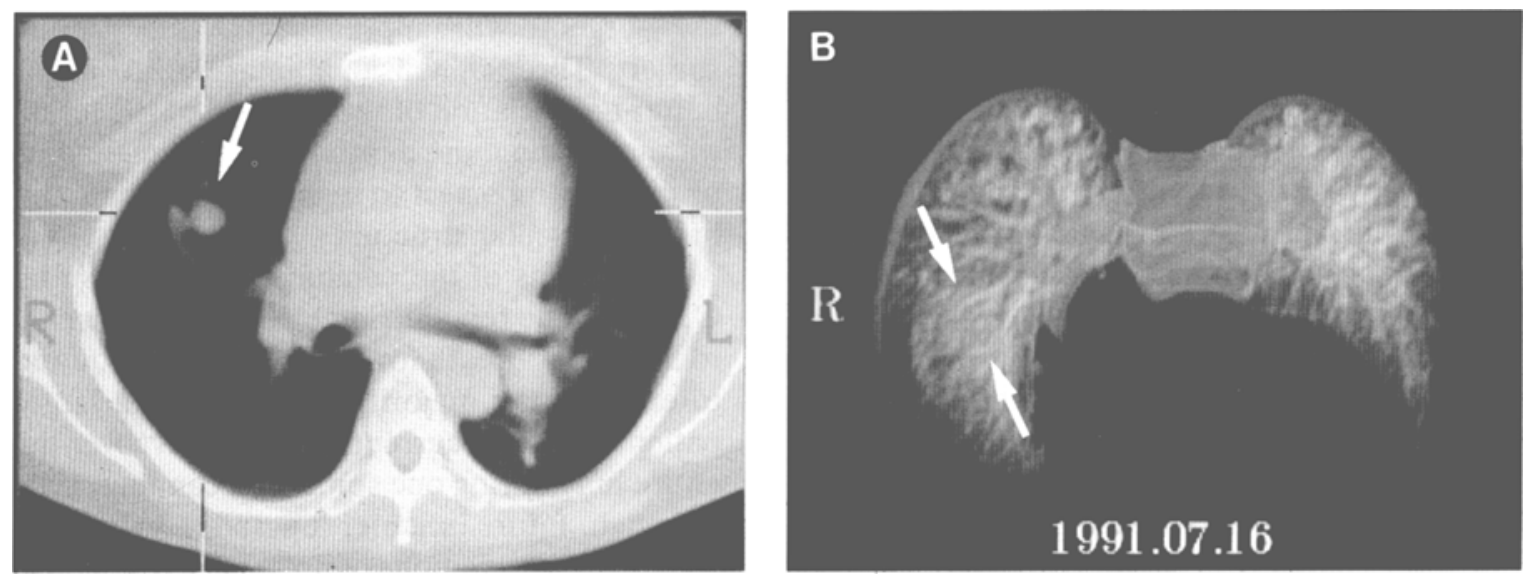

Fig 3. Pulmonary arteriovenous malformation. (A) Axial view generated from spiral CT dataset shows a nodule with a feeding artery and draining vein compatible with a pulmonary arteriovenous malformation (AVM) (arrow). (B) Volumetric rendering of the lung using spiral $C T$ data sets shows pulmonary AVM in a 3-D format (arrows).

$8 \mathrm{~mm}$, table speed $8 \mathrm{~mm} / \mathrm{sec}$, and slice reconstruction every $4 \mathrm{~mm}$.)

\section{Case 3}

A 36-year-old woman with a history of fallopian tube cancer was examined to exclude

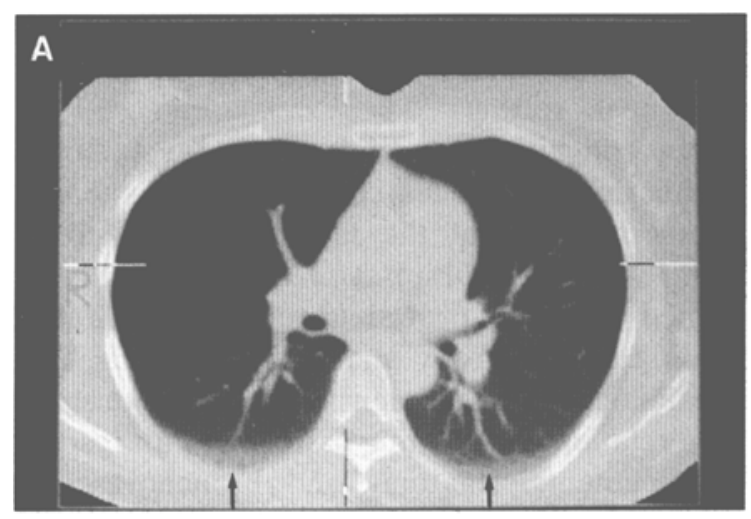

Fig 4. Dependent edema. \{A \} Axial CT scan shows dependent edema in the posterior aspects of both lower lungs (arrows). Multiplanar reconstructed image in the sagittal plane $(B)$, as well as the 3-D images of the lung $(C)$ accurately reproduce the findings of dependent edema (arrows). On the sagittal reconstructed image $(B)$, one can appreciate that the entire lower lobe is of increased density compared with the upper lobe farrow. heads). The right and left main stem bronchi are displayed on the 3-D image $(C)$. (Viewer's perspective of the lungs in the 3-D image $(C)$ is from above, looking down onto the lungs.) pulmonary metastases. No lung metastases were identified on the CT, but the study does show dependent edema in the posterior aspects of both lower lungs (Fig 4A), a normal finding. Multiplanar reconstructed images in the sagittal plane (Fig 4B), as well as the 3-D image of the
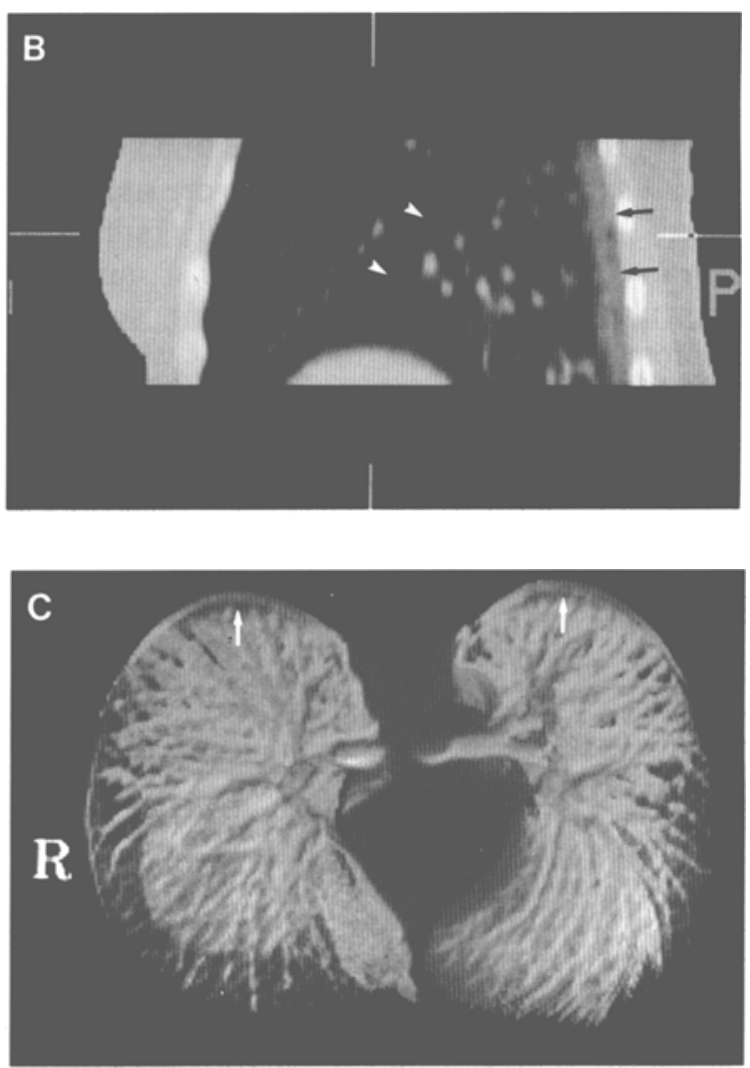
lung (Fig 4C) accurately reproduce the findings of dependent edema. On the sagittal reconstructed image (Fig 4B), one can appreciate that the entire lower lobe is of increased density compared with the upper lobe. (Protocol 1: slice acquisition thickness $8 \mathrm{~mm}$, table speed $8 \mathrm{~mm} /$ $\mathrm{sec}$, and slice reconstruction every $4 \mathrm{~mm}$.)

\section{DISCUSSION}

Most current 3-D imaging techniques have been applied primarily to orthopedics and craniofacial abnormalities..$^{8-11}$ We began exploring the feasibility of 3-D imaging of the lung by applying volumetric rendering techniques, first to inflated-fixed-lung specimens. From this work, we were able to develop a modified classification scheme that allows faithful depiction of lung structures. ${ }^{12}$ The next challenge was to apply or adapt these techniques to the lungs of a living, breathing patient. The use of singlebreath-hold spiral CT datasets that allow reformation of images with little motion degradation has made this now possible.

This combined 2-D/3-D spiral CT technique has a number of potentially useful clinical applications. Three-dimensional modeling of the trachea and major airways provides a more detailed anatomic guide for the bronchoscopist or surgeon. Volumetric quantification of lung disease could be used to perform more sophisticated 3-D tumor volumetrics or 3-D radiation therapy planning. Three-dimensional imaging may also prove useful for planning surgical approaches in patients undergoing wedge or segmental resection of primary or metastatic tumors in the lung. One of the advantages of the technique is the ability to display lung pathology in a 3-D format that more closely resembles how the surgeon will approach the lung during surgery. Three-dimensional imaging provides a better appreciation of the location of the lesion within the lung, the depth of the lesion, and its position with respect to segmental anatomy and bronchovascular structures.

Limitations of the combined 2-D/3-D spiral CT technique remain. Some patients are unable to hold their breath for the 24 seconds required to examine the majority of the lung. In larger patients, it is not currently possible to cover the entire lung during one spiral CT scanning cycle, although future scanner upgrades will allow spiral data acquisitions of up to 60 seconds.

Radiation dose from a spiral CT is equivalent to that from a standard CT acquisition that uses the same slice collimation thickness, mA-sec, and $\mathrm{kV}$ (peak) settings. A spiral $\mathrm{CT}$ with a slice collimation thickness $X$ equal to the table feed distance of $X$ per scan is equivalent in dose to a standard CT with a section acquisition thickness $\mathrm{S}$ equal to $\mathrm{X}$. For example, a spiral $\mathrm{CT}$ scan using protocol 1 with slice acquisition thickness $8 \mathrm{~mm}$, table speed $8 \mathrm{~mm} / \mathrm{sec}$, and slice reconstruction every $4 \mathrm{~mm}$ would be equivalent to a standard CT using contiguous $8 \mathrm{~mm}$ thick slices, 1-second scan times, and the same mA-sec and $\mathrm{kV}$ (peak) settings.

In conclusion, multiplanar and volumetric rendering techniques create accurate, nondegraded images of the lung when applied to single-breath-hold spiral CT datasets. The combined technique proved advantageous in delineating major airway anatomy, displaying lung pathology in three dimensions, and demonstrating relationships of lung pathology with respect to segmental anatomy and bronchovascular structures.

\section{REFERENCES}

1. Kalender WA, Seissler W, Klotz E, et al: Spiral volumetric CT with single-breath-hold technique, continuous transport, and continuous scanner rotation. Radiology 176:181-183, 1990

2. Shaffer K, Pugatch RD: Small pulmonary nodules: Dynamic CT with a single-breath technique. Radiology 173:567-568, 1989

3. Vock P, Soucek M, Daepp M, et al: Lung: Spiral volumetric $C T$ with single-breath-hold technique. Radiology 176:864-867, 1990

4. Costello P, Anderson W, Blume D: Pulmonary nodule: Evaluation with spiral volumetric CT. Radiology 179:875876,1991
5. Fishman EK, Drebin RA, Magid D, et al: Volumetric rendering techniques: Applications for three-dimensional imaging of the hip. Radiology 163:737-738, 1987

6. Fishman EK, Drebin RA, Hruban RH, et al: Threedimensional reconstruction of the human body. AJR Am J Roentgenol 150:1419-1420, 1988

7. Ney DR, Fishman EK, Magid D, et al: Interactive real-time multiplanar CT imaging. Radiology 170:275-276, 1989

8. Vannier MW, Totty WG, Stevens WG, et al: Musculoskeletal applications of three-dimensional surface reconstruction. Orthop Clin North Am 16:543-555, 1985

9. Pate D, Resnick D, Andre M, et al: Perspective: 
Three-dimensional imaging of the musculoskeletal system. AJR Am J Roentgenol 147:545-551, 1986

10. Vannier MW, Marsh JL, Warren JO: Three-dimensional $\mathrm{CT}$ reconstruction images for craniofacial surgical planning and evaluation. Radiology 150:179-184, 1984

11. Fishman EK, Magid D, Ney DR, et al: Three- dimensional CT imaging in orthopedics; State of the ART 1988. Orthopedics 2:1021-1026, 1986

12. Ney DR, Kuhlman JE, Hruban RH, et al: Threedimensional CT-volumetric reconstruction and display of the bronchial tree. Invest Radiol 25:736-742, 1990 